\title{
Drivers of Brand Page Attachment: An Abstract
}

\author{
Rico Piehler, Michael Schade, Barbara Kleine-Kalmer, \\ and Christoph Burmann
}

\begin{abstract}
While there are behavior-related constructs like brand engagement that measure the success of activities in social networks, a psychological, pre-behavioral construct is missing in literature. Therefore, brand page attachment as strength of the bond connecting the brand page with the self of the user is introduced. Based on an empirical study with 590 German Facebook users, brand page attachment is revealed as strong predictor of behavior like brand page participation (like, comment, and share posts). Concerning the drivers of brand page attachment, social value had the largest effect, followed by infotainment and economic incentives. Based on these results, brand page attachment should be considered as psychological, pre-behavioral construct in social media research and practice.
\end{abstract}

References Available Upon Request

R. Piehler $(\bowtie) \bullet$ M. Schade $\bullet$ B. Kleine-Kalmer $\bullet$ C. Burmann University of Bremen, Bremen, Germany

e-mail: rico.piehler@uni-bremen.de; mschade@uni-bremen.de; limsekr@uni-bremen.de;

burmann@uni-bremen.de 